\title{
An optimization approach for a joint location inventory model considering quantity discount policy
}

\begin{abstract}
Joint location inventory problems involve determination of two key decisions in a supply chain network: inventory control decisions and facility location decisions. This paper addresses a supply chain network consisting of a supplier and a set of retailers, in which a number of distribution centers (DCs) are to be located. The objectives of a joint location inventory problem are to identify the optimal number and location of the DCs, the assignment of the retailers to the DCs and determining the inventory control decision of the DCs so that the total cost is minimized. This paper extends the traditional joint location inventory model that considers the basic EOQ policy to replenish the inventory of retailers, by considering quantity discount as the inventory policy of the supply chain network. The new model can be applied to both EOQ and quantity discount policies. An efficient two-stage heuristic algorithm is developed to solve the model. Numerical experiments show the efficiency of the proposed algorithm.
\end{abstract}

Keyword: Location inventory problem; Distribution network design; Quantity discount; EOQ model; Heuristic algorithm; Simulated annealing 